\title{
Efficient Numerical Solution of Steady Free-Surface Navier-Stokes Flow
}

\author{
E. H. van Brummelen, ${ }^{*} .1$ H. C. Raven,$\dagger$ and B. Koren* \\ ${ }^{*}$ CWI, P.O. Box 94079, 1090 GB Amsterdam, The Netherlands; and †Maritime Research \\ Institute Netherlands, P.O. Box 28,6700 AA Wageningen. The Netherlands \\ E-mail: harald.van.brummelen@cwi.nl; h.c.raven@marin.nl; barry.koren@cwi.nl
}

Received March 1, 2001; revised July 6, 2001

\begin{abstract}
Numerical solution of flows that are partially bounded by a freely moving boundary is of great importance in practical applications such as ship hydrodynamics. The usual method for solving steady viscous free-surface flow subject to gravitation is alternating time integration of the kinematic condition, and the NavierStokes equations subject to the dynamic conditions, until steady state is reached. This paper shows that this time integration approach is often inefficient. It proposes an efficient iterative method for solving the steady free-surface flow problem. The new method relies on a different but equivalent formulation of the free-surface flow problem, involving a so-called quasi free-surface condition. The convergence behavior of the new method is shown to be asymptotically mesh-width independent. Numerical results are presented for two-dimensional flow over an obstacle in a channel. The results confirm the mesh-width independence of the convergence behavior, and comparison of the numerical results with measurements shows good agreement. (c) 2001 Elsevier Science

Key Words: numerical solution methods; free-surface flows; incompressible NavierStokes equations.
\end{abstract}

\section{INTRODUCTION}

The numerical solution of flows that are partially bounded by a freely moving boundary is of great importance in ship hydrodynamics $[1,6,8,13]$, hydraulics, and many other practical applications, such as coating technology $[16,17]$. In ship hydrodynamics, an important area of application is the prediction of the wave pattern that is generated by the ship at forward speed in still water. This wave generation is responsible for a substantial part of the ship's resistance, and therefore, it should be minimized by a proper hull form design. Computational

\footnotetext{
' Research supported by Maritime Research Institute Netherlands (MARIN).
} 
methods play an important role in this design process. Most computational tools that are currently in use for solving gravity-subjected free-surface flows around a surface-piercing body rely on a potential flow approximation. Present developments primarily concern the solution of the free-surface Navier-Stokes (or RANS) flow problem.

For time-dependent free-surface flows, generally there is no essential difference in the treatment of the free surface between numerical methods for potential flow or NavierStokes flow. Typically, the solution of the flow equations and the adaptation of the free boundary are separated. Each time step begins with computing the flow field using the dynamic conditions imposed at the free surface. Next, the free surface is adjusted through the kinematic condition, employing the newly computed velocity field.

For steady free-surface flows, however, such a conformity of approaches for viscous and inviscid flow cannot be observed. For instance in ship hydrodynamics, whereas dedicated techniques have been developed for solving the free-surface potential flow problems (see, e.g., [15]), methods for Navier-Stokes flow usually continue the aforementioned transient process until a steady state is reached (see, e.g., $[1,6])$. However, this time integration method is often computationally inefficient. In general, the convergence to steady state is retarded by slowly attenuating transient surface-gravity waves. Moreover, the separate treatment of the flow equations and the kinematic condition yields a restriction on the allowable time step. Owing to the specific transient behavior of free-surface flows and the time-step restriction, the performance of the time integration method deteriorates rapidly with decreasing mesh width. In practical computations, tens of thousands of time steps are often required, rendering the time integration approach prohibitively expensive in actual design processes.

Several approaches have been suggested to improve the efficiency of time-integration methods (e.g., pseudo-time integration [8] and quasi-steady methods [22]). It appears that these approaches indeed improve the efficiency, but do not essentially improve the asymptotic convergence behavior of the time-integration method.

Alternative solution methods for steady free-surface Navier-Stokes flow exist, but they have not been widely applied in the field of ship hydrodynamics. In the field of coating technology successive approximation techniques are often employed, in particular kinematic iteration and dynamic iteration [17]. Kinematic iteration imposes the dynamic conditions at the free surface and uses the kinematic condition to displace the boundary. Dynamic iteration imposes the kinematic and the tangential dynamic conditions at the free surface and uses the normal dynamic condition to adjust the boundary position. However, the convergence behavior of both iteration schemes depends sensitively on parameters in the problem (see, e.g., $[5,19]$ ). A method that avoids the deficiencies of the aforementioned iterative methods, is Newton iteration of the full equation set [17]. The positions of the (freesurface) grid nodes are then added as additional unknowns and all equations, including the free-surface conditions, are solved simultaneously. An objection to this method is that simultaneous treatment of all equations is infeasible for problems with many unknowns, such as three-dimensional problems and problems requiring sharp resolution of boundary layers. Finally, the free-surface flow problem can be reformulated into an optimal-shape design problem, which can then in principle be solved efficiently by the adjoint optimization method. A problem with this approach is its complexity: although much progress has been made in the formulation of adjoint equations for problems from fluid dynamics, including the Navier-Stokes equations [9], setting up the adjoint method remains involved. Moreover, efficiency is only obtained if proper preconditioning is applied [20,21], and constructing the preconditioner for the free-surface Navier-Stokes flow problem is intricate. 
The current work presents an iterative method for efficiently solving steady free-surface Navier-Stokes flow problems. Although our interest is the previously outlined ship hydrodynamics application, it is anticipated that the method is also applicable to other gravitydominated steady viscous free-surface flows at high Reynolds numbers, such as occur, for instance, in hydraulics. The proposed method is analogous to the method for solving steady free-surface potential flow problems presented in Ref. [15]. The method alternatingly solves the steady Navier-Stokes equations with a so-called quasi free-surface condition imposed at the free surface and adjusts the free surface using the computed solution. The quasi freesurface condition ensures that the disturbance induced by the subsequent displacement of the boundary is negligible. Each surface adjustment then yields an improved approximation to the actual free-boundary position.

The contents of the paper are organized as follows. In Section 2 the equations governing incompressible, viscous free-surface flow are stated and the quasi free-surface condition is derived. Section 3 proves that the usual time integration approach is generally inept for solving steady free-surface flows. Section 4 outlines the iterative solution method and examines its convergence behavior. Numerical experiments and results for a two-dimensional test case are presented in Section 5. The application to actual ship-wave computations is in progress and will be reported in a sequel. Section 6 contains concluding remarks.

\section{GOVERNING EQUATIONS}

\subsection{Incompressible Viscous Flow}

An incompressible, viscous fluid flow subject to a constant gravitational force is considered. Although only steady solutions are of interest, for the purpose of analysis the equations are considered in time-dependent form.

The fluid occupies an open, time-dependent domain $\mathcal{V}_{\eta} \subset \mathbb{R}^{d}(d=2,3)$, which is enclosed by the free boundary, $\mathcal{S}_{\eta}$, and a fixed boundary, $\partial \mathcal{V}_{\eta} \backslash \mathcal{S}_{\eta}$. Positions in $\mathbb{R}^{d}$ are identified by their horizontal coordinates $\left(x_{1}, \ldots, x_{d-1}\right)$ and a vertical coordinate $y$, with respect to the Cartesian base vectors $\mathbf{e}_{1}, \ldots, \mathbf{e}_{d-1}$ and $\mathbf{j}$, respectively. The origin is located in the undisturbed free surface $\mathcal{S}_{0}$, and the gravitational acceleration, $\mathbf{g}$, acts in the negative vertical direction. We consider free surfaces that can be represented by a so-called height-function, i.e., $\mathcal{S}_{\eta}=\{(\mathbf{x}, \eta(\mathbf{x}, t))\}$. The height-function $\eta$ is assumed to be a smooth function of the horizontal coordinates and time (see Fig. 1 for an illustration).

The distinguishing parameters of the viscous free-surface flow problem are the Froude number, Fr $\equiv U / \sqrt{g \ell}$, and the Reynolds number, Re $\equiv \rho U \ell / \mu$, with $U$ an appropriate

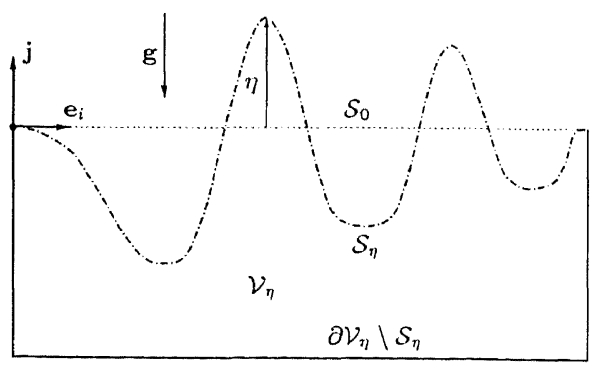

FIG. 1. Schematic illustration of the free-surface flow problem. 
reference velocity, $g$ the gravitational acceleration, $\ell$ a reference length, and $\mu$ the dynamic viscosity of the fluid. The fluid density $\rho$ is assumed to be constant. The state of the flow is then characterized by the (nondimensionalized) fluid velocity $\mathbf{v}(\mathbf{x}, y, t)$ and pressure $p(\mathbf{x}, y, t)$. Incompressibility of the fluid implies that the velocity field is solenoidal:

$$
\operatorname{div} \mathbf{v}=0, \quad(\mathbf{x}, y) \in \mathcal{V}_{\eta}, t>0 .
$$

Conservation of momentum in the fluid is described by the Navier-Stokes equations. The pressure is separated into a hydrodynamic component $\varphi$ and a hydrostatic contribution as $p(\mathbf{x}, y, t)=\varphi(\mathbf{x}, y, t)-\mathrm{Fr}^{-2} y$. Because the gradient of the hydrostatic pressure and the gravitational force cancel, the Navier-Stokes equations for a gravity-subjected incompressible fluid read

$$
\frac{\partial \mathbf{v}}{\partial t}+\operatorname{div} \mathbf{v v}+\nabla \varphi-\operatorname{div} \tau(\mathbf{v})=0, \quad(\mathbf{x}, y) \in \mathcal{V}_{\eta}, t>0,
$$

where $\tau(\mathbf{v})$ is the viscous stress tensor for an incompressible Newtonian fluid,

$$
\tau(\mathbf{v})=\operatorname{Re}^{-1}\left((\nabla \mathbf{v})+(\nabla \mathbf{v})^{T}\right) .
$$

Our primary interest is in turbulent flows. We consider the Reynolds averaged NavierStokes (RANS) equations, supplemented with a turbulence model that is based on eddy viscosity. For our purpose, the RANS equations are essentially the same as the NavierStokes equations, with the important difference being that the RANS equations have steady solutions even at the envisaged high Reynolds numbers.

\subsection{Free-Surface Conditions}

Free-surface flows are essentially two-phase flows, of which the properties of the contiguous bulk fluids are such that their mutual interaction at the interface can be ignored. For an elaborate discussion of two-phase flows, see, for example, Refs. [2] and [18]. The free-surface conditions follow from the general interface conditions and the assumptions that both density and viscosity of the adjacent fluid vanish at the interface and, furthermore, that the interface is impermeable. Here it is moreover assumed that interfacial stresses can be ignored, which is a valid assumption in the practical applications envisaged.

On the free surface, the fluid satisfies a kinematic condition and $d$ dynamic conditions. Impermeability of the free surface is expressed by the kinematic condition

$$
\frac{\partial \eta}{\partial t}+\mathbf{v} \cdot \nabla(\eta-y)=0, \quad(\mathbf{x}, y) \in \mathcal{S}_{\eta}, t>0 .
$$

Supposing that the viscous contribution to the normal stress at the free surface is negligible, continuity of stresses at the interface requires that the pressure vanish at the free surface. This results in the normal dynamic condition

$$
\varphi-\operatorname{Fr}^{-2} \eta=0, \quad(\mathbf{x}, y) \in \mathcal{S}_{\eta}, t>0 .
$$

The requirement that the tangential stress components vanish at the free surface is expressed by the $d-1$ tangential dynamic conditions

$$
\mathbf{t}^{i} \cdot \tau(\mathbf{v}) \cdot \mathbf{n}=0, \quad(\mathbf{x}, y) \in \mathcal{S}_{\eta}, t>0 .
$$


Here, $\mathbf{t}^{i}(i=1, \ldots, d-1)$ are orthogonal unit tangent vectors to $\mathcal{S}_{\eta}$, and $\mathbf{n}$ denotes the unit normal vector to $\mathcal{S}_{\eta}$.

One may note that the number of free-surface conditions for the viscous free-surface flow problem is $d+1$. The incompressible Navier-Stokes equations in $\mathbb{R}^{d}$ require $d$ boundary conditions. Hence, the number of free-surface conditions is indeed one more than the number of required boundary conditions.

\subsection{Quasi Free-Surface Condition}

A fundamental problem in analyzing and computing free-surface flow problems is the interdependence of the state variables $\mathbf{v}$ and $p$ and their spatial domain of definition through the free-surface conditions. This problem can be avoided by deriving a condition that holds to good approximation on a fixed boundary in the neighborhood of the actual free boundary. We refer to such a condition as a quasi free-surface condition, because the qualitative solution behavior of the initial boundary value problem with this condition imposed is similar to that of the free-boundary problem, but the boundary does not actually move. A suitable quasi free-surface condition for the free-surface Navier-Stokes flow problem is derived below.

Let $\mathcal{S}_{\eta}$ denote the actual free surface, as defined before. In a similar manner, a nearby fixed boundary $\mathcal{S}_{\theta}=\{(\mathbf{x}, \theta(\mathbf{x}))\}$ is introduced, with $\theta(\mathbf{x})$ a smooth function on $\mathcal{S}_{()}$. We require that $\mathcal{S}_{\theta}$ be close to the actual free surface in such a manner that

$$
\delta(\mathbf{x}, t) \equiv \eta(\mathbf{x}, t)-\theta(\mathbf{x})
$$

is small and sufficiently smooth. In particular, for all $t>0, \delta$ must satisfy $\|\delta\|_{\mathcal{S}_{1 \prime}}+\|\nabla \delta\|_{\mathcal{S}^{\prime \prime}}+$ $\left\|\delta_{t}\right\|_{\mathcal{S}_{H}} \leq \epsilon$, for some $\epsilon \ll 1$. Here $\|\cdot\|_{\mathcal{S}_{H}}$ is a suitable norm on the approximate boundary. Assuming that $p$ and $\mathbf{v}$ can be extended smoothly beyond the boundary $\mathcal{S}_{H}$, Taylor expansion in the neighborhood of $\mathcal{S}_{\theta}$ yields for $p$ and $v$ at the actual free surface

$$
\begin{aligned}
& p(\mathbf{x}, \eta(\mathbf{x}, t), t)=p(\mathbf{x}, \theta(\mathbf{x}), t)+\delta(\mathbf{x}, t) \mathbf{j} \cdot \nabla p(\mathbf{x}, \theta(\mathbf{x}), t)+O\left(\epsilon^{2}\right) \\
& \mathbf{v}(\mathbf{x}, \eta(\mathbf{x}, t), t)=\mathbf{v}(\mathbf{x}, \theta(\mathbf{x}), t)+\delta(\mathbf{x}, t) \mathbf{j} \cdot \nabla \mathbf{v}(\mathbf{x}, \theta(\mathbf{x}), t)+O\left(\epsilon^{2}\right)
\end{aligned}
$$

The normal dynamic condition (2b) demands that the left-hand side of Eq. (4a) vanish. Hence, the elevation of the free surface can be expressed in terms of the pressure and its gradient at the approximate surface:

$$
\eta(\mathbf{x}, t)=\theta(\mathbf{x})-\frac{p(\mathbf{x}, \theta(\mathbf{x}), t)}{\mathbf{j} \cdot \nabla p(\mathbf{x}, \theta(\mathbf{x}), t)}+O\left(\epsilon^{2}\right) .
$$

To obtain an $O\left(\epsilon^{2}\right)$ accurate quasi free-surface condition (i.e., an $O\left(\epsilon^{2}\right)$ approximation of the conditions at $\mathcal{S}_{\theta}$ ), $\eta$ and $v$ in the kinematic condition (2a) can be replaced by Eqs. (5) and (4b), respectively. The resulting condition is, however, intractable. Instead, two additional assumptions concerning $\mathbf{v}$ and $p$ are introduced to obtain a convenient quasi free-surface condition. The first assumption is that the vertical derivative of the pressure is dominated by the hydrostatic component, $-\mathrm{Fr}^{-2}$. Generally, this assumption is valid for waves of moderate steepness. Specifically, we suppose that a constant $\sigma_{p} \ll 1$ exists such that for all $t>0$,

$$
\left\|1+\operatorname{Fr}^{2} \mathbf{j} \cdot \nabla p\right\|_{\mathcal{S}_{H}} \leq \sigma_{p}
$$


The second assumption is that the vertical derivative of $\mathbf{v}$ is small, in such a manner that a constant $\sigma_{\mathrm{v}} \ll 1$ exists with the property that, for all $t>0$,

$$
\|\mathbf{j} \cdot \nabla \mathbf{v}\|_{\mathcal{S}_{H}} \leq \sigma_{v} .
$$

Under this assumption, the velocity at the actual free boundary, $\mathbf{v}(\mathbf{x}, \eta(\mathbf{x}, t), t)$, can be accurately approximated by the velocity at the fixed boundary, $\mathbf{v}(\mathbf{x}, \theta(\mathbf{x}), t)$. By Eq. (4b), the error in the approximation is only $O\left(\epsilon \sigma_{\mathbf{v}}\right)$. In Ref. [3] it is shown that the velocity deviation through the free-surface boundary layer is proportional to the surface curvature and $1 / \sqrt{\mathrm{Re}}$. Moreover, $\sigma_{v}$ in Eq. (7) increases with the wave steepness. Therefore, the assumption $\sigma_{v} \ll 1$ is valid if the steepness and curvature of the free-surface waves are moderate and if $\operatorname{Re}$ is sufficiently large.

Under the above assumptions a convenient quasi free-surface condition can be derived. Substitution of the hydrostatic approximation of the pressure gradient in Eq. (5) yields

$$
\eta(\mathbf{x}, t)=\theta(\mathbf{x})-\frac{p(\mathbf{x}, \theta(\mathbf{x}), t)}{-\mathrm{Fr}^{-2}\left(1+O\left(\sigma_{p}\right)\right)}=\theta(\mathbf{x})+\operatorname{Fr}^{2} p(\mathbf{x}, \theta(\mathbf{x}), t)\left(1+O\left(\sigma_{p}\right)\right) .
$$

The dynamic conditions (2b) and (4a) imply that $p=O(\epsilon)$ on $\mathcal{S}_{\theta}$. Hence, ignoring terms $O\left(\epsilon^{2}, \epsilon \sigma_{p}\right)$, the free-surface elevation is related to the hydrodynamic pressure at the approximate boundary by

$$
\eta(\mathbf{x}, t)=\theta(\mathbf{x})+\operatorname{Fr}^{2} p(\mathbf{x}, \theta(\mathbf{x}), t)=\operatorname{Fr}^{2} \varphi(\mathbf{x}, \theta(\mathbf{x}), t) .
$$

To transfer the kinematic condition ( $2 a$ ) to the approximate surface $\mathcal{S}_{\theta}, \eta$ is replaced by Eq. (9) and $\mathbf{v}$ on $\mathcal{S}_{\eta}$ is replaced by $\mathbf{v}$ on $\mathcal{S}_{\theta}$. The error thus introduced is only $O\left(\epsilon^{2}, \epsilon \sigma_{p}, \epsilon \sigma_{\mathbf{v}}\right)$. Special care is required in expressing the gradient of $\eta$, because Eq. (9) relates $\eta$ to $\varphi$ on the curvilinear surface $\mathcal{S}_{H}$ :

$$
\nabla \eta=\operatorname{Fr}^{2} \frac{d \varphi}{d \mathbf{x}}=\operatorname{Fr}^{2}\left(\frac{\partial \varphi}{\partial \mathbf{x}}+\frac{\partial \varphi}{\partial y} \frac{\partial \theta}{\partial \mathbf{x}}\right)=\operatorname{Fr}^{2}\left(\nabla \varphi+\frac{\partial \varphi}{\partial y}\left(\frac{\partial \theta}{\partial \mathbf{x}}-\mathbf{j}\right)\right) .
$$

It then follows that

$$
\begin{aligned}
\frac{\partial \eta}{\partial t}+\mathbf{v} \cdot \nabla(\eta-y)= & \operatorname{Fr}^{2}\left(\frac{\partial \varphi}{\partial t}+\mathbf{v} \cdot \nabla\left(\varphi-\mathrm{Fr}^{-2} y\right)\right) \\
& +\operatorname{Fr}^{2} \frac{\partial \varphi}{\partial y} \mathbf{v} \cdot\left(\frac{\partial \theta}{\partial \mathbf{x}}-\mathbf{j}\right)+O\left(\epsilon^{2}, \epsilon \sigma_{p}, \epsilon \sigma_{\mathbf{v}}\right)=0
\end{aligned}
$$

Using the kinematic condition (2a) and definition (3), the second term on the right-hand side of Eq. (11) can be recast as

$$
\operatorname{Fr}^{2} \frac{\partial \varphi}{\partial y} \mathbf{v} \cdot \nabla(\theta-y)=\operatorname{Fr}^{2} \frac{\partial \varphi}{\partial y} \mathbf{v} \cdot \nabla(\eta-\delta-y)=-\operatorname{Fr}^{2} \frac{\partial \varphi}{\partial y}\left(\mathbf{v} \cdot \nabla \delta+\delta_{t}\right) .
$$

Owing to the smoothness of $\delta$, the term in parenthesis is just $O(\epsilon)$ and Eq. (12) is only $O\left(\epsilon \sigma_{p}\right)$. The second term on the right-hand side of Eq. (11) can therefore be ignored. Hence, it follows that

$$
\frac{\partial \varphi}{\partial t}+\mathbf{v} \cdot \nabla\left(\varphi-\operatorname{Fr}^{-2} y\right)=0
$$


approximates the conditions at the boundary $\mathcal{S}_{H}$ to $O\left(\epsilon^{2}, \epsilon \sigma_{p}, \epsilon \sigma_{\mathrm{v}}\right)$. This implies that Eq. (13) is a quasi free-surface condition on any fixed boundary that is sufficiently close to the actual free surface, provided that Eqs. (6) and (7) are fulfilled.

One may note that Eq. (13) is exactly satisfied at the actual free surface. Therefore, the quasi free-surface condition can replace either the kinematic condition (2a) or the normal dynamic condition (2b) in the formulation of the free-surface conditions in Section 2.2.

The importance of the quasi free-surface condition is that the quasi free-surface flow solution (i.e., the solution of the Navier-Stokes equations with Eqs. (13) and (2c) imposed at a fixed boundary in the neighborhood of the actual free surface) is an accurate approximation of the actual free-surface flow solution. Because the tangential dynamic conditions are largely irrelevant to the shape of the free surface [3], it is anticipated that the change in the solution due to imposing Eq. (2c) at $\mathcal{S}_{H}$ instead of $\mathcal{S}_{\eta \mid}$ is negligible. In that case, if Eq. (13) holds at $\mathcal{S}_{H}$, then the free surface conditions (2b) and (2a) are satisfied to $O\left(\epsilon^{2}, \epsilon \sigma_{p}, \epsilon \sigma_{\mathbf{v}}\right)$ at the boundary

$$
\left\{\left(\mathbf{x}, \operatorname{Fr}^{2} \varphi(\mathbf{x}, \theta(\mathbf{x}, t))\right)\right\} .
$$

Therefore, the solution of the quasi free-surface flow problem is an $O\left(\epsilon^{2}, \epsilon \sigma_{p}, \epsilon \sigma_{\mathbf{v}}\right)$ approximation to the solution of the free-surface flow problem. Moreover, (14) is an equally accurate approximation of the actual free-surface position. One may note that (14) just uses the normal dynamic condition to determine the position of the free surface.

\section{TIME INTEGRATION METHODS}

The most widely applied iterative method for solving gravity-dominated steady freesurface Navier-Stokes flow is alternating time integration of the kinematic condition, and the Navier-Stokes equations subject to the dynamic conditions, until steady state is reached. This section examines the computational complexity of this time integration method (i.e., the number of operations per grid point expended in the solution process).

The computational complexity of the time integration method depends on the physical time that is required to reduce transient wave components in the initial estimate to the level of other errors in the numerical solution. The transient behavior of surface gravity waves therefore plays an essential part in the complexity analysis. This transient behavior is discussed in Sections 3.1 and 3.2. Next, the implications on the computational complexity are examined in Section 3.3.

\subsection{Surface Gravity Waves}

We consider the specific case of a small-amplitude disturbance of a uniform horizontal flow on a domain $\mathcal{V} \subset \mathbb{R}^{d}$ of infinite horizontal extent and unit vertical extent. The domain is bounded by the undisturbed free surface $\mathcal{S}_{0}=\{(\mathbf{x}, 0)\}$ and a rigid impermeable free-slip bottom $\mathcal{B}=\{(\mathbf{x},-1)\}$. The uniform flow velocity is $\mathbf{v}^{(0)}=\left(v_{1}^{(0)}, \ldots, v_{d-1}^{(0)}, 0\right)$, with $\left|v^{(0)}\right|=1$. The above implies that the undisturbed fluid depth and flow velocity are designated as reference length and velocity, respectively.

Suppose that a disturbance is generated in the flow, such that for all $t>0$ the resulting surface-elevation satisfies $\|\eta\|_{\mathcal{S}_{01}}+\|\nabla \eta\|_{\mathcal{S}_{11}}+\left\|\eta_{t}\right\|_{\mathcal{S}_{11}} \leq \epsilon$, for some positive $\epsilon$. We assume 
that the corresponding perturbed free-surface flow solution can be written as

$$
\left(\begin{array}{l}
\mathbf{v} \\
\varphi
\end{array}\right)(\mathbf{x}, y, t ; \epsilon)=\left(\begin{array}{c}
\mathbf{v}^{(0)} \\
0
\end{array}\right)+\epsilon\left(\begin{array}{c}
\mathbf{v}^{(1)} \\
\varphi^{(1)}
\end{array}\right)(\mathbf{x}, y, t)+O\left(\epsilon^{2}\right), \quad \text { as } \epsilon \rightarrow 0 .
$$

From Section 2.3 it follows that the solution of the quasi free-surface flow problem on $\mathcal{V}$ is an $O\left(\epsilon^{2}, \epsilon \sigma_{p}, \epsilon \sigma_{\mathrm{v}}\right)$ approximation of the actual free-surface flow, with $\sigma_{p}$ and $\sigma_{\mathrm{v}}$ defined by Eqs. (6) and (7), respectively. However, Eq. (15) implies that $\sigma_{p}$ and $\sigma_{\mathrm{v}}$ are of $O(\epsilon)$. Hence, the quasi free-surface flow solution on $\mathcal{V}$ is an $O\left(\epsilon^{2}\right)$ approximation to the actual free-surface flow solution. Consequently, for sufficiently small and smooth perturbations the results on the behavior of the quasi free-surface flow solution apply immediately to the behavior of the actual free-surface flow solution.

Suppose that the disturbance can be written as a linear combination of horizontal Fourier modes $\exp (\mathrm{ik} \cdot \mathbf{x}+\mathrm{i} \omega t)$, with $\mathbf{k} \in \mathbb{R}^{d-1}$ the wavenumber of the Fourier mode and $\omega$ its frequency. Because the perturbed quasi free-surface flow problem is linear to $O\left(\epsilon^{2}\right)$, it suffices to consider a single mode. If the following Fourier mode is inserted for the perturbations in Eq. (15),

$$
\left(\begin{array}{c}
v_{1}^{(1)} \\
\vdots \\
v_{d-1}^{(1)} \\
v_{d}^{(1)} \\
\varphi^{(1)}
\end{array}\right)(\mathbf{x}, y, t)=\left(\begin{array}{c}
\mathrm{i} k_{1} \cosh (|\mathbf{k}|(1+y)) \\
\vdots \\
\mathrm{i} k_{d-1} \cosh (|\mathbf{k}|(1+y)) \\
|\mathbf{k}| \sinh (|\mathbf{k}|(1+y)) \\
(-1)^{j} \mathrm{i} \Phi(\mathbf{k}) \cosh (|\mathbf{k}|(1+y))
\end{array}\right) \exp \left(\mathrm{ik} \cdot \mathbf{x}+\mathrm{i} \omega_{j}(\mathbf{k}) t\right),
$$

where $\omega_{j}(\mathbf{k})$ is either of the two roots of the dispersion relation,

$$
\omega_{j}(\mathbf{k})=-\mathbf{v}^{(0)} \cdot \mathbf{k}-(-1)^{j} \Phi(\mathbf{k}), \quad j=1,2,
$$

and

$$
\Phi(\mathbf{k})=\sqrt{\mathrm{Fr}^{-2}|\mathbf{k}| \tanh (|\mathbf{k}|)},
$$

then the corresponding $\mathbf{v}$ and $\varphi$ comply to $O\left(\epsilon^{2}\right)$ with the quasi free-surface flow problem, except for the tangential dynamic conditions (2c), which yield

$$
\mathbf{t}^{i} \cdot \tau(\mathbf{v}) \cdot \mathbf{n}=\operatorname{Re}^{-1} \epsilon 2 \mathrm{i} k_{j}|\mathbf{k}| \sinh (|\mathbf{k}|) \exp \left(\mathrm{ik} \cdot \mathbf{x}+\mathrm{i} \omega_{j}(\mathbf{k}) t\right) .
$$

Because Eq. (17) is only $O\left(\epsilon|\mathbf{k}|^{3} / \operatorname{Re}\right)$ as $|\mathbf{k}| \rightarrow 0$, the error is negligible for sufficiently small $\mathbf{k}$ and large Re. Hence, Eq. (16a) accurately describes the behavior of smooth free-surface waves in a uniform horizontal flow at sufficiently high Reynolds numbers. The perturbations (16a) are called surface gravity waves. (For an elaborate discussion of surface gravity waves in potential flow see, e.g., Refs. [11, 12]).

\subsection{Asymptotic Temporal Behavior}

The asymptotic temporal behavior of surface gravity waves is determined by the asymptotic properties of the Fourier integral of the modes (16a). The behavior of the integral 
transform for $t \rightarrow \infty$ can be determined by means of the asymptotic expansion

$$
\begin{aligned}
& \int_{0}^{\infty} F(k) \exp (\mathbf{i} t \psi(k)) d k \\
& \quad=F\left(k_{0}\right) \sqrt{\frac{2 \pi}{t\left|\psi^{\prime \prime}\left(k_{0}\right)\right|}} \exp \left(\mathrm{i}\left[t \psi\left(k_{0}\right)+\frac{1}{4} \pi \operatorname{sign} \psi^{\prime \prime}\left(k_{0}\right)\right]\right)+O\left(e^{-\beta t}\right),
\end{aligned}
$$

with $\beta$ a positive constant, $F(k)$ an analytic function, and $k_{0}$ a stationary point of $\psi(k)$ (i.e., $\psi^{\prime}\left(k_{0}\right)=0$ ). The expansion (18) requires that $\psi(k)$ be smooth in the neighborhood of stationary points in the sense that the ratio $\psi^{\prime \prime \prime}\left(k_{0}\right) /\left|\psi^{\prime \prime}\left(k_{0}\right)\right|^{3 / 2}$ is small (see Ref. [11]). The method of stationary phase (sometimes called the method of steepest descent) can be used to prove (18) (see, e.g., Refs. [12, 25]).

The Fourier integral of Eq. (16a) can be evaluated for $t \rightarrow \infty$ by introducing a suitable coordinate transformation for $\mathbf{k}$ and applying Eq. (18) recursively with respect to the transformed coordinates. Denoting by $\sigma(\mathbf{x}, y, t)$ a component in Eq. (16a) and by $\hat{\sigma}(\mathbf{k}, y)$ its Fourier transform, one obtains

$\sigma(\mathbf{x}, y, t)=\hat{\sigma}\left(\mathbf{k}_{0}, y\right)(2 \pi / t)^{(d-1) / 2}\left(\operatorname{det} \mathbf{H}\left(\mathbf{k}_{0}\right)\right)^{-1 / 2} \exp \left(\mathrm{i} t \psi\left(\mathbf{k}_{0}\right)+\mathrm{i} \zeta\right)+O\left(e^{-\beta t}\right)$

as $t \rightarrow \infty$, where

$$
\psi(\mathbf{k})=\mathbf{k} \cdot \mathbf{x} / t+\omega_{\alpha}(\mathbf{k})
$$

and where $\mathbf{H}(\mathbf{k})$ denotes its Hessian and $\zeta$ is a multiple of $\pi / 4$ depending on the properties of the Hessian (see also Ref. [24]). By Eqs. (16b) and (16c), for fixed $\mathbf{x}$ and $t \rightarrow \infty$, a stationary point $\mathbf{k}_{0}$ of $\psi(\mathbf{k})$ occurs when

$$
\frac{\partial \Phi(\mathbf{k})}{\partial k_{j}}=\operatorname{Fr}^{-1} \frac{\tanh |\mathbf{k}|+|\mathbf{k}|\left(1-\tanh ^{2}|\mathbf{k}|\right)}{2 \sqrt{|\mathbf{k}| \tanh |\mathbf{k}|}} \frac{k_{j}}{|\mathbf{k}|}=v_{j}^{(0)}, \quad j=1, \ldots, d-1 .
$$

Assuming that $\mathbf{v}^{(0)}$ is scaled such that $\left|\mathbf{v}^{(0)}\right|=1$, a sufficient and necessary condition for a stationary point to exist is $\operatorname{Fr}^{-2} \Lambda(|\mathbf{k}|)=1$, with

$$
\Lambda(|\mathbf{k}|)=\frac{\left(\tanh |\mathbf{k}|+|\mathbf{k}|\left(1-\tanh ^{2}|\mathbf{k}|\right)\right)^{2}}{4|\mathbf{k}| \tanh |\mathbf{k}|}
$$

One can show that $\Lambda(|\mathbf{k}|)$ is a bijection from $\mathbb{R}_{+}$to $(0,1]$. Therefore, a single stationary point exists if and only if Fr $\leq 1$ (i.e., for subcritical flows). This stationary point corresponds to a wave of which the group velocity (see, e.g., Refs. [12, 24]) equals the flow velocity. Consequently, the energy associated with this wave remains at a fixed position and decays only owing to dispersion.

By Eq. (19a), at subcritical Froude numbers the asymptotic temporal behavior of the surface-gravity waves (16) in $\mathbb{R}^{d}$ is $O\left(t^{(1-d) / 2}\right)$ as $t \rightarrow \infty$. In particular, surface gravity waves attenuate as $1 / \sqrt{t}$ in $\mathbb{R}^{2}$ and as $1 / t$ in $\mathbb{R}^{3}$. At supercritical Froude numbers, a stationary point of $\psi(\mathbf{k})$ does not exist and the first term in Eq. (19a) disappears. The surface gravity waves then vanish exponentially as $t \rightarrow \infty$. 


\subsection{Computational Complexity}

Suppose the objective is to solve a steady free-surface flow problem using the time integration method. The asymptotic temporal behavior of surface gravity waves can then be used to estimate the asymptotic computational complexity of the method.

Spatial discretization of the incompressible Navier-Stokes equations with appropriate boundary conditions on fixed boundaries and the free-surface conditions on the free boundary yields a discrete operator $\mathbf{L}_{h}: \mathcal{A}_{h} \mapsto \mathcal{B}_{h}$, with $\mathcal{A}_{h}$ denoting the space of grid functions on a grid with characteristic mesh-width $h$. The operator $\mathbf{L}_{h}$ is assumed to be stable and $p$ th order consistent (i.e., the discretization error, $\epsilon_{h}$, is $O\left(h^{p}\right)$ as $h \rightarrow 0$ ).

Numerical time integration of the spatially discretized free-surface flow problem yields a sequence $\mathbf{q}_{h}^{\prime \prime} \in \mathcal{A}_{h}, n=0,1,2, \ldots$. The grid-function $\mathbf{q}_{h}^{0}$ is a restriction of initial conditions to the grid. Assuming the time step in the time integration method, $\tau$, to be constant, $\mathbf{q}_{h}^{n}$ approximates the solution of the free-surface flow problem at time $t=n \tau$. Suppose that the discretized free-surface flow problem has a unique solution $\mathbf{q}_{h}^{*} \in \mathcal{A}_{h}$, and that the sequence $\mathbf{q}_{h / h}^{n}$ indeed approaches $\mathbf{q}_{h}^{*}$ as $n \tau \rightarrow \infty$. The evaluation error is defined by

$$
\gamma^{n}=\left\|q_{h}^{n}-q_{h}^{*}\right\| .
$$

If the aim is to approximate the solution of the steady free-surface flow problem, it is sufficient to reduce the evaluation error to the level of the discretization error. Further reduction does not yield an essential improvement in the approximation of the continuum solution anyway. By (19a), the asymptotic behavior of the evaluation error at subcritical Froude numbers is

$$
\gamma^{n}=O\left((n \tau)^{(1-d) / 2}\right), \quad \text { as } n \tau \rightarrow \infty .
$$

For an example of this convergence behavior in actual computations, see the numerical experiments on fine grids in Ref. [22]. From Eq. (23) it follows that $\gamma^{\prime \prime} \leq \epsilon_{h}$ requires

$$
n=O\left(h^{2 p /(1-l)} \tau^{-1}\right), \quad \text { as } h \rightarrow 0 .
$$

Equation (24) implies an increase of the number of time steps to reach steady state within the required tolerance. This is particularly manifest for high-order discretizations (large $p$ ) and low spatial dimension $(d=2)$.

An additional complication is that usually the allowable time step decreases with $h$. Time integration of free-surface flow problems typically proceeds in two alternating steps: (T1) integrate the incompressible Navier-Stokes, subject to the dynamic conditions at the free surface and appropriate boundary conditions at fixed boundaries; and (T2) integrate the kinematic condition to adjust the free-surface position, using the solution from (T1).

Owing to this separate treatment and the hyperbolic character of the kinematic condition, stability of the numerical time integration method requires that the time step comply with a CFL condition, $\tau \propto h$.

In summary, Eq. (24) and the CFL condition imply that the number of time steps required to reach $\gamma^{n} \leq \epsilon_{h}$ is $O\left(h^{-(1+2 p /(d-1) \prime)}\right)$. Assuming that the computational complexity of the time integration method is proportional to the number of time steps, at subcritical Froude numbers the computational complexity is

$$
W=O\left(h^{-(1+2 p /(d-1))}\right), \quad \text { as } h \rightarrow 0 .
$$


Equation (25) implies a severe increase in the computational expenses as $h$ decreases. For example, in the typical case of a second-order discretization of the three-dimensional problem, if the mesh width is halved, the required computational work per grid point increases by a factor of 8 .

\section{EFFICIENT SOLUTION OF STEADY FREE-SURFACE FLOWS}

From Section 3 it is evident that the usual time integration approach is inept for solving steady free-surface flows at subcritical Froude numbers. In this section we present an efficient iterative solution method for gravity-subjected steady free-surface flows. The method is outlined in Section 4.1. The convergence properties of the method and its computational complexity are examined in Sections 4.2 and 4.3 .

\subsection{Iterative Solution Method}

From the results in Section 2.3, it follows that an accurate approximation to the freesurface flow and to the free-surface position can be obtained by the following operations.

(I1) For a given initial boundary $\mathcal{S}$, solve $(\mathbf{v}, \varphi)$ from

$$
\begin{aligned}
& \left.\begin{array}{rl}
\operatorname{div} \mathbf{v} \mathbf{v}+\nabla \varphi-\operatorname{div} \tau(\mathbf{v}) & =0 \\
\operatorname{div} \mathbf{v} & =0
\end{array}\right\}, \quad(\mathbf{x}, y) \in \mathcal{V}, \\
& \mathbf{B}(\mathbf{v}, p)=\mathbf{b}(\mathbf{x}, y), \quad(\mathbf{x}, y) \in \dot{\nu} \backslash \mathcal{S}, \\
& \left.\begin{array}{r}
\mathbf{t}^{i} \cdot \tau(\mathbf{v}) \cdot \mathbf{n}=0 \\
\mathbf{v} \cdot \nabla \varphi-\mathrm{Fr}^{-2} \mathbf{j} \cdot \mathbf{v}=0
\end{array}\right\}, \quad(\mathbf{x}, y) \in \mathcal{S},
\end{aligned}
$$

where Eq. (26b) represents boundary conditions on the fixed boundary.

(I2) Use the solution of (I1) to adjust the boundary $\mathcal{S}$ to

$$
\left\{\left(\mathbf{x}, y+\operatorname{Fr}^{2} \varphi(\mathbf{x}, y)\right):(\mathbf{x}, y) \in \mathcal{S}\right\} .
$$

Note the appearance of the quasi free-surface condition in its steady form in Eq. (26c). The modified boundary approximates the actual free surface more accurately than does the initial boundary, provided that the conditions discussed in Section 2.3 are fulfilled. Hence, it is anticipated that the solution to the free-surface flow problem can be obtained by iterating the operations (I1) and (I2).

If $\mathcal{S}$ is the actual free surface, then the normal dynamic condition is satisfied (i.e., $p$ vanishes on $\mathcal{S}$ ). In that case, $\mathbf{n} \| \nabla p$, and Eq. (26c) implies that the solution of Eqs. (26) complies with the kinematic condition and the tangential dynamic conditions. Hence, operation (I1) then yields the free-surface flow. Moreover, the normal dynamic condition ensures that the surface adjustment in (I2) vanishes, so that the solution of the free-surface flow problem is indeed a fixed point of the iteration.

It is important to notice the absence of time-dependent terms in (I1) and (I2). Therefore, the slow decay of transient waves described in Section 3 is irrelevant to the convergence of the iterative process. The actual convergence properties of (I1)-(I2) are examined below. 


\subsection{Convergence}

The convergence behavior of the iterative method (I1)-(I2) can be conveniently examined by rephrasing the free-surface flow problem as an optimal-shape design problem. A general characteristic of free-boundary problems is that the number of free-boundary conditions is one more than the number of boundary conditions required by the governing boundary-value problem. A free-boundary problem can therefore be reformulated into the equivalent optimal-shape design problem of finding the boundary that minimizes a norm of the residual of one of the free-surface conditions, subject to the boundary-value problem with the remaining free-surface conditions imposed.

To obtain an optimal-shape design formulation of the steady free-surface flow problem, the cost functional $E$ is defined by

$$
E(\mathcal{S},(\mathbf{v}, p)) \equiv \int_{\mathcal{S}}|p(\mathbf{x}, y)| \mathrm{d} \mathcal{S} .
$$

Assuming that Eq. (26) is well posed for all surfaces $\mathcal{S}$ in a space of admissible boundaries $\mathcal{O}$, and that $\mathcal{O}$ contains the actual free surface, the free-surface flow problem is equivalent to the optimal-shape design problem

$$
\min _{\mathcal{S} \in \mathcal{O}}\{E(\mathcal{S},(\mathbf{v}, p)):(\mathbf{v}, p) \text { satisfies }(26)\}
$$

Notice that problem (29) is in fact a constrained optimization problem, with the boundary value problem (26) serving as a constraint on $(\mathbf{v}, p)$.

The optimal-shape design formulation of the free-surface flow problem allows convenient assessment of the convergence properties of the iterative method (I1)-(I2). Each iteration adjusts the approximation to the free-surface position. Convergence of the iterative method is ensured if each surface adjustment yields a reduction in the cost functional (28). Moreover, the reduction of the cost functional between successive iteractions is a measure of the efficiency of the method.

To determine the effect of a surface adjustment, consider the boundary $\mathcal{S}$ and the modified boundary

$$
\mathcal{S}_{\epsilon \alpha}=\{(\mathbf{x}, y)+\epsilon \alpha(\mathbf{x}, y) \mathbf{j}:(\mathbf{x}, y) \in S\},
$$

for a suitably smooth function $\alpha$ independent of $\epsilon$ on $\mathcal{S}$. The modified boundary is the boundary of a domain $\mathcal{V}_{\epsilon \alpha}$, which approaches $\mathcal{V}$ as $\epsilon \rightarrow 0$. Following Ref. [14], $\mathcal{V}$ and $\mathcal{V}_{\epsilon \alpha}$ are embedded in a bounded set $\varepsilon$ and it is assumed that for all $\mathcal{V} \subset \varepsilon$ with $\mathcal{S} \in \mathcal{O}$, a solution for Eq. (26) can be extended smoothly beyond the boundary, so that $(\mathbf{v}, p)$ is well defined everywhere in $\varepsilon$.

The displacement of the boundary from $\mathcal{S}$ to $\mathcal{S}_{\epsilon \alpha}$ induces a disturbance in the solution of Eq. (26). Denoting by $(\mathbf{v}, p)_{\epsilon \alpha}$ the solution of Eq. (26) on $\mathcal{V}_{\epsilon \alpha}$, the induced disturbance is defined by

$$
(\mathbf{v}, p)_{\alpha}^{\prime} \equiv \lim _{\epsilon \rightarrow 0} \frac{1}{\epsilon}\left((\mathbf{v}, p)_{\epsilon \alpha}-(\mathbf{v}, p)\right)
$$

Taylor expansion of the cost functional then yields

$$
E\left(\mathcal{S}_{\epsilon \alpha},(\mathbf{v}, p)_{\epsilon \alpha}\right)=\int_{\mathcal{S}}\left|p+\epsilon\left(\alpha \mathbf{j} \cdot \nabla p+p_{\alpha}^{\prime}\right)\right|\left(1+\epsilon \mu_{\alpha}\right) \mathrm{d} \mathcal{S}+O\left(\epsilon^{2}\right), \quad \text { as } \epsilon \rightarrow 0 .
$$


In Eq. (32), the function $\mu_{\alpha}: \mathcal{S} \mapsto \mathbb{R}$ accounts for the change in the surface area from d $\mathcal{S}$ to $\mathrm{d} \mathcal{S}_{\epsilon \alpha}$. Ignoring terms $O\left(\epsilon^{2}\right)$, the modified boundary $\mathcal{S}_{\epsilon \alpha}$ improves on $\mathcal{S}$ if a positive constant $\zeta<1$ exists such that

$$
\int_{\mathcal{S}}\left|p+\epsilon\left(\alpha \mathbf{j} \cdot \nabla p+p_{\alpha}^{\prime}\right)\right|\left(1+\epsilon \mu_{\alpha}\right) \mathrm{d} \mathcal{S} \leq \zeta \int_{\mathcal{S}}|p| \mathrm{d} \mathcal{S} .
$$

If condition (33) holds for some $\zeta<1$, then the modification of the boundary from $\mathcal{S}$ to $\mathcal{S}_{\epsilon \alpha}$ yields a reduction in the cost functional. The smallest positive constant that satisfies condition (33) is called the contraction number. Clearly, a small contraction number implies a successful surface modification.

Operation (I2) in the iterative procedure gives a correction in the boundary position $\epsilon \alpha=\mathrm{Fr}^{2} p$. In that case, the value of the cost functional corresponding to the modified surface is bounded by

$$
E\left(\mathcal{S}_{\epsilon \alpha},(\mathbf{v}, p)_{\epsilon \alpha}\right) \leq \int_{\mathcal{S}}|p|\left|1+\operatorname{Fr}^{2} \mathbf{j} \cdot \nabla p\right|\left(1+\epsilon \mu_{\alpha}\right) \mathrm{d} \mathcal{S}+\int_{\mathcal{S}}\left|\epsilon p_{\alpha}^{\prime}\right| \mathrm{d} \mathcal{S} .
$$

Hence, the contraction number $\zeta$ of the iterative process (I1)-(I2) is bounded by

$$
\zeta \leq \sigma_{p}+\frac{\int_{\mathcal{S}}\left|\epsilon p_{\alpha}^{\prime}\right| \mathrm{d} \mathcal{S}}{\int_{\mathcal{S}}|p| \mathrm{d} \mathcal{S}}+O(\epsilon)
$$

with $\sigma_{p}$ defined by Eq. (6). From condition (35) it follows that if $\epsilon$ and $\sigma_{p}$ are indeed small, then the induced disturbance determines the convergence behavior of the iterative method.

To establish convergence of (I1)-(I2), it remains to be seen whether the induced disturbance $p_{\alpha}^{\prime}$ is indeed small. In Section 2.3 it was shown that the quasi free-surface condition (13) approximates the conditions at a fixed boundary in the neighborhood of the free surface to $O\left(\epsilon^{2}, \epsilon \sigma_{p}, \epsilon \sigma_{\mathrm{v}}\right)$. Hence, displacing this condition from $\mathcal{S}$ to $\mathcal{S}_{\epsilon \alpha}$ yields no greater disturbance than that. In Ref. [3] it is shown that the tangential dynamic conditions are largely irrelevant to the shape of the free surface. Conversely, the induced disturbance due to enforcing the tangential dynamic conditions at $\mathcal{S}$ instead of $\mathcal{S}_{\epsilon \alpha}$ can be neglected. Therefore, the contraction number of the iterative method (I1)-(I2) is estimated as

$$
\zeta=O\left(\epsilon, \sigma_{p}, \sigma_{v}\right)
$$

\subsection{Computational Complexity}

Equation (36) provides an upper bound for the contraction number of the iterative method (I1)-(I2). One may note that if the approximate boundary is sufficiently close to the actual free surface ( $\epsilon$ small), then Eq. (36) depends on properties of the continuum solution only. Therefore, if the free-surface flow problem is solved numerically, the convergence behavior of the iterative method is asymptotically independent of mesh width.

The iteration must be continued until the pressure defect at the free surface (28) has been reduced to the level of the spatial discretization error. Further reduction does not essentially improve the approximation of the continuum solution anyway. Each iteration reduces the pressure defect at the free surface by a factor $\zeta$. Therefore, the number of iterations $n$ must satisfy

$$
\zeta^{n}=O\left(h^{p}\right)
$$


This implies that $n=O(p \log h / \log \zeta)$. Assuming that the computational complexity of the iterative method is proportional to the number of iterations, the following estimate of the computational complexity is obtained:

$$
W=O(\log h)
$$

Hence, the efficiency of the iterative method (I1)-(I2) deteriorates only moderately with decreasing mesh width.

To eliminate the remaining weak $h$-dependence of the computational complexity, nested iteration can be employed. Generally, an iterative solution method is used to solve the boundary-value problem (26) in step (I1) of the algorithm. The nesting involves the use of the solution from the previous iteration as an initial estimate for the solution process. Because this initial estimate becomes increasingly accurate, the cost of performing (I1) reduces as the iteration progresses. In particular, assuming that the cost of solving Eq. (26) is proportional to the pressure defect at the free surface, the amount of work that is required to achieve Eq. (37) is

$$
W=w+\zeta w+\zeta^{2} w+\cdots+\zeta^{n} w \leq \frac{1}{1-\zeta} w
$$

with $w$ denoting the cost of solving Eq. (26) initially. Observe that the computational complexity (39) is indeed entirely independent of the mesh width.

\section{NUMERICAL EXPERIMENTS AND RESULTS}

The method is tested for subcritical flow over an obstacle in a channel of unit depth, at $\mathrm{Fr}=0.43$ and $\mathrm{Re}=1.5 \times 10^{5}$, with the undisturbed fluid depth and the undisturbed flow velocity at the free surface assigned as the reference length and velocity, respectively. The geometry of the obstacle is

$$
y(x)=-1+\frac{27}{4} \frac{H}{L^{3}} x(x-L)^{2}, \quad 0 \leq x \leq L,
$$

with $H$ and $L$ the (nondimensionalized) height and length of the obstacle, respectively. Choosing $H=0.2$ and $L=2$, the setup is in agreement with that in Ref. [5]. At the bottom boundary no-slip boundary conditions are imposed. A boundary-layer velocity profile in accordance with the experiments from Ref. [5] is imposed at the inflow boundary.

The test case with $H=0.2$ displays large-amplitude waves that exhibit typical nonlinear effects, such as sharp wave crests and wavelength reduction. In addition, $H=0.15$ is considered. This test case displays waves more in accordance with linear wave theory (see, e.g., Refs. $[11,12])$.

The experiments are performed on grids with horizontal mesh widths $h=2^{-5}, 2^{-6}$. The number of grid cells in the vertical direction is 70 and exponential grid stretching is applied to resolve the boundary layer at the bottom. Furthermore, the grid is coarsened toward the inflow and outflow boundaries to reduce reflections. A typical example of a grid used in the numerical experiments is presented in Fig. 2. The RANS equations, closed with an eddyviscosity model owing to Cebeci and Smith [7], and the boundary conditions are discretized and solved by the method described in Ref. [10]. After each evaluation, the grid is adapted using vertical stretching. An initial estimate of the solution on the adapted grid is subsequently generated by linear interpolation from the solution on the previous grid. Details of the discretization method and the setup of the numerical experiments can be found in Ref. [4]. 


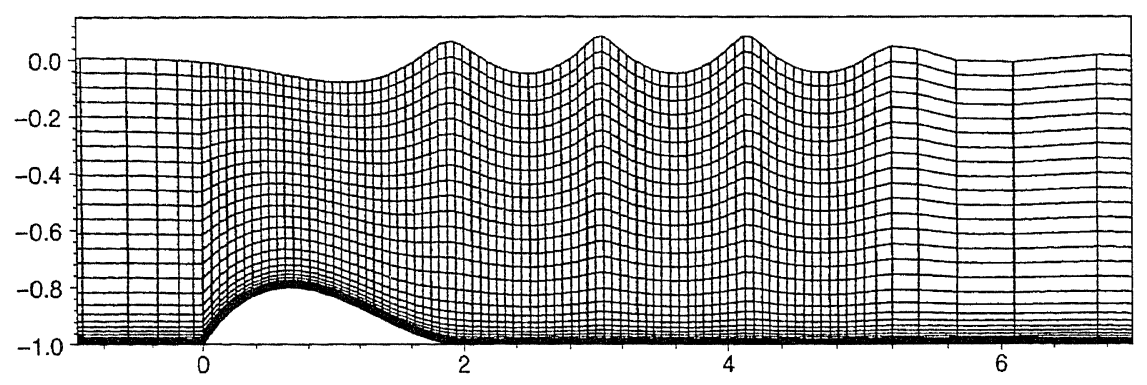

FIG. 2. Example of a grid used in the numerical experiments. The grid is coarsened for illustration purposes.

Figure 3 shows the wave profile obtained in successive iterations for $H=0.2$. The initial estimate (zeroth iterate) is just the undisturbed free surface. One may note that the first iterate already displays a qualitatively correct wave profile. This confirms that the quasi free-surface flow solution is an accurate approximation of the actual free-surface flow solution. A converged solution is obtained in less than 10 iterations. Owing to the decreasing computational cost of each iteration (refer to Section 4.3), the entire computation is just two to three times as expensive as the corresponding fixed domain problem with symmetry boundary conditions at the undisturbed surface.

Figure 4 displays the pressure defect at the free surface after consecutive iterations. The results confirm convergence of the method. For $H=0.15$, the average contraction number is $\zeta \approx 0.15$ and the convergence behavior is indeed independent of $h$. After several iterations the contraction number increases. However, this is entirely due to the fact that the quasi free-surface flow problem (26) is solved only by approximation. If the tolerance on the residual of Eq. (26) is reduced (i.e., if Eq. (26) is solved more accurately), then the original contraction number is recovered. For $H=0.20$, the average contraction number is $\zeta \approx 0.45$ for $h=2^{-5}$ and $\zeta \approx 0.52$ for $h=2^{-6}$. As a result of strong nonlinearity, the asymptotic mesh-width independence of the convergence behavior is in this case not yet apparent.

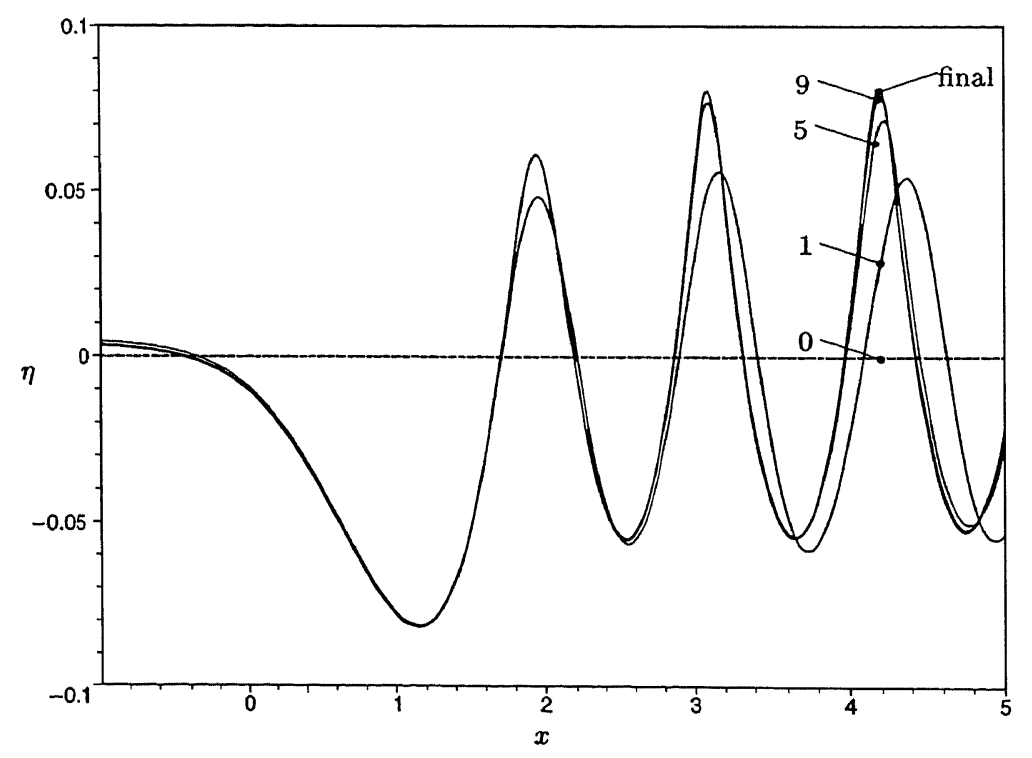

FIG. 3. Wave profile obtained after successive iterations $(H=0.2)$. 


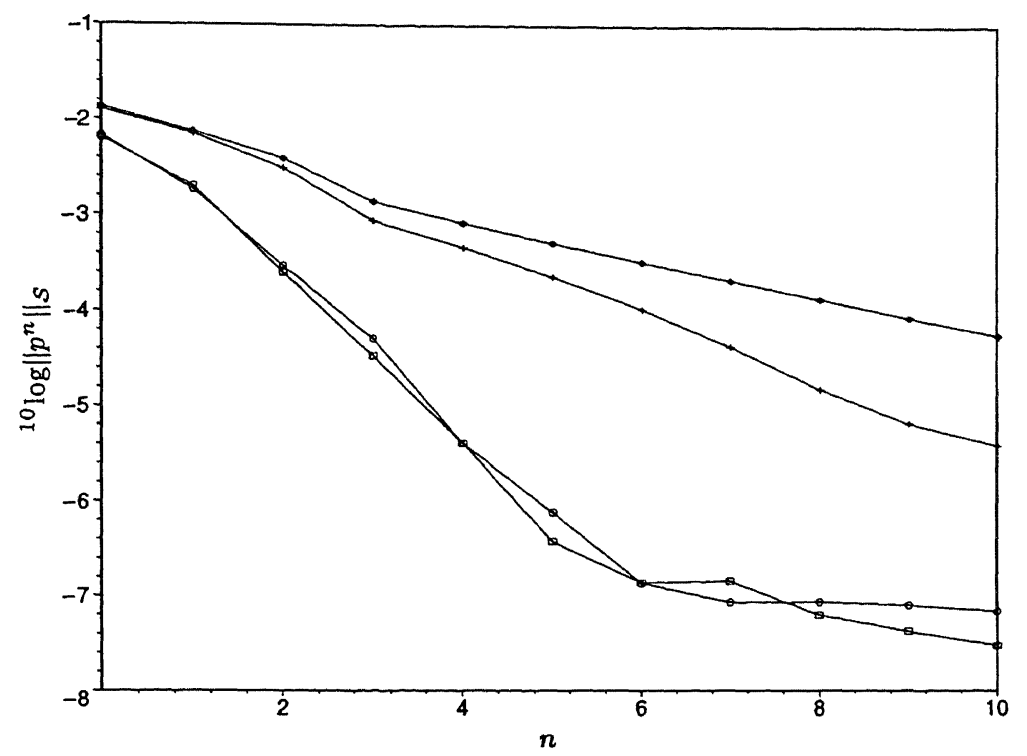

FIG. 4. Pressure defect at the free surface versus the iteration number for $H=0.15 . h=2^{-5}(\square), h=2^{-h}(O)$, and $H=0.20, h=2^{-5}(+), h=2^{-5}(\diamond)$.

A detailed investigation of the convergence behavior of time integration methods for the test case with $H=0.20$ is presented in Ref. [22]. Typically, the time integration method requires approximately $10^{4}$ surface adjustments to reduce the initial error by a factor of 10 . The presented method achieves this in approximately four iterations, for a similar setting of the numerical experiment.

Figure 5 compares the computed wave elevation with measurements from Ref. [5]. In Ref. [5], a nondimensionalized amplitude $a=4.5 \times 10^{-2} \pm 15 \%$ and wavelength

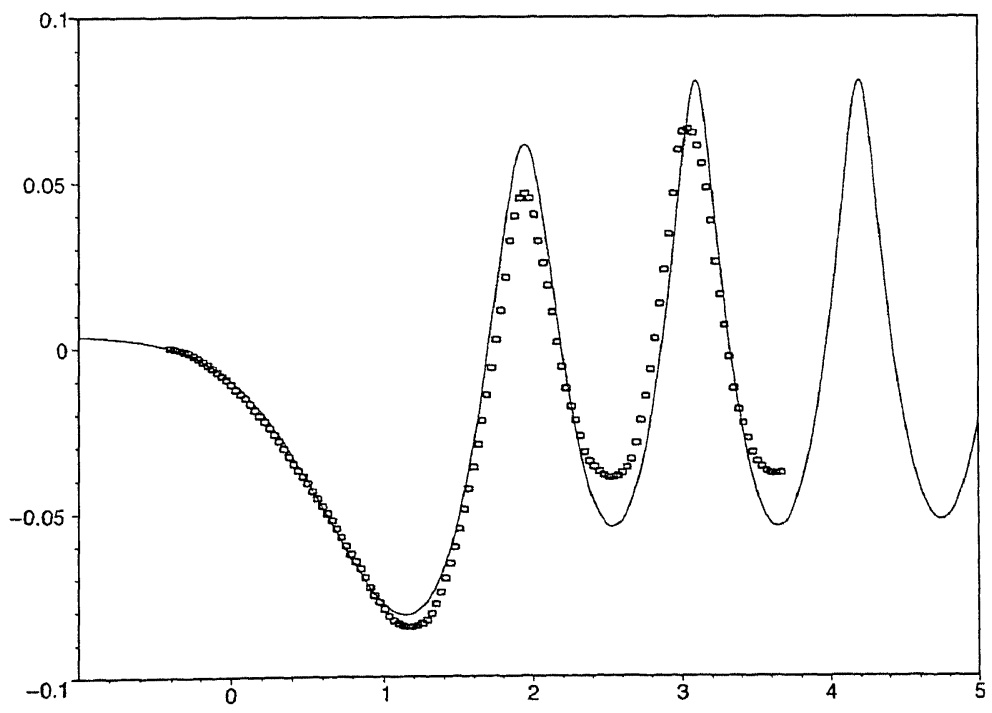

FIG. 5. Computed wave elevation for $h=2^{-6}$ (solid line) and measurements from Ref. [5] (markers only), for $H=0.20$. The obstacle is located in the interval $x \in[0,2]$. 
$\lambda=1.10 \pm 10 \%$ are reported for the trailing wave. The trailing wave of the computed wave elevation on the grid with $h=2^{-6}$ displays amplitude $a=6.5 \times 10^{-2}$ and wavelength $\lambda=1.11$. Hence, the computed wavelength agrees well with the measurements. The amplitude appears to be overestimated. However, the difference between the amplitude of the numerical results and of the experimental data is not unusual (see, e.g., Refs. [22, 23]). Observe also that the difference in the amplitude of the first wave and the second wave is correctly predicted.

\section{CONCLUSION}

The usual time-integration method for solving steady free-surface Navier-Stokes flow problems was shown to be inefficient owing to the specific transient behavior of surfacegravity waves and a CFL condition on the allowable time step.

Motivated by the demand for efficient computational methods in practical applications, we proposed a new iterative-solution method. The method alternatingly solves the steady Navier-Stokes equations with a quasi free-surface condition imposed at the free surface, and adjusts the free surface using the computed solution and the normal dynamic condition.

Examination of the convergence properties of the iterative method revealed that the method uses the quasi free-surface condition to ensure that the disturbance induced by the displacement of the boundary is small. It was shown that the convergence behavior of the method is asymptotically independent of the mesh width. The asymptotic computational complexity of the iterative method deteriorates only moderately with decreasing mesh width. Mesh-width independence of the computational complexity can be achieved by means of nested iteration.

Numerical results were presented for two-dimensional flow over an obstacle in a channel. For the presented test cases, a converged solution was obtained in at most 10 iterations. The numerical results agree well with measurements. The numerical experiments confirmed that the convergence behavior of the method is asymptotically independent of mesh width.

We believe that the proposed method will be useful in ship hydrodynamics, hydraulics, and other fields of application in which the efficient computation of steady free-surface flows at high Reynolds number is required.

\section{REFERENCES}

1. B. Alessandrini and G. Delhommeau, Simulation of three-dimensional unsteady viscous free surface flow around a ship model, Int. J. Num. Meth. Fluids 19, 321 (1994).

2. R. Aris, Vectors, Tensors and the Basic Equations of Fluid Mechanics (Prentice Hall, Englewood Cliffs, NJ, 1962).

3. G. K. Batchelor, An Introduction to Fluid Dynamics (Cambridge Univ. Press, Cambridge, UK, 1967).

4. E. H. van Brummelen, Numerical solution of steady free-surface Navier-Stokes flow, Technical Report MAS-R0018, ISSN 1386-3703, CWI, 2000. Available at http://www.cwi.nl/ftp/CWIreports/MAS/ MAS-R0018.ps.Z.

5. J. Cahouet, Etude Numérique et Experimentale du Problème Bidimensionnel de la Résistance de Vague's Non-Linéaire, Ph.D. thesis (ENSTA, Paris, 1984).

6. E. Campana, A. Di Mascio, P. G. Esposito, and F. Lalli, Viscous-inviscid coupling in free surface ship flows, Int. J. Num. Meth. Fluids 21, 699 (1995).

7. T. Cebeci and A. M. O. Smith, Analysis of Turbulent Boundary Layers (Academic, New York, 1974). 
8. J. Farmer, L. Martinelli, and A. Jameson, A fast multigrid method for solving the nonlinear ship wave problem with a free surface, in Proceedings of the 6th International Conference on Numerical Ship Hydrodynamics, Iowa 1993, edited by Patel and Stern (National Academy Press, Washington D.C., 1994), p. 1.55.

9. M. B. Giles and N. A. Pierce, Adjoint equations in CFD: Duality, boundary conditions and solution behaviour, AIAA Paper 97, 1850 (1997).

10. M. Hoekstra, Numerical Simulation of Ship Stern Flow's with a Space-Marching Navier-Stokes Method, Ph.D. thesis (Delft University of Technology, Netherlands, 1999).

11. H. Lamb, Hydrodynamics, 6th ed. (Dover, NY, 1945).

12. M. J. Lighthill, Waves in Fluids (Cambridge Univ. Press, Cambridge, 1978).

13. H. Miyata, T. Sato, and N. Babo, Difference solution of a viscous flow with free-surface wave about an advancing ship, J. Comput. Phys. 72, 393 (1987).

14. O. Pironneau, Optimal Shape Design for Elliptic Systems (Springer-Verlag, Berlin, 1984).

15. H. C. Raven, A Solution Method for the Nonlinear Ship Wave Resistance Problem, Ph.D. thesis (Delft Univ. Technol., Netherlands, 1996).

16. P. A. Sackinger, P. R. Schuck, and R. R. Rao, A Newton-Raphson pseudo-solid domain mapping technique for free and moving boundary problems: A finite element implementation, J. Comput. Phys. 125, 83 (1996).

17. H. Saito and L. E. Scriven, Study of coating flow by the finite element method, J. Comput. Phys. 42, 53 (1981).

18. L. E. Scriven, Dynamics of a fluid interface, Chem. Eng. Sci. 12, 98 (1960).

19. W. J. Silliman and L. E. Scriven, Separating flow near a static contact line: Slip at a wall and shape of a free surface, J. Comput. Phys. 34, 287 (1980).

20. S. Ta'asan, Infinite dimensional preconditioners for optimal design problems, in Inverse Design and Optimization Methods, edited by R. A. van den Braembussche and M. Manna, VKI Lecture Series (Von Karman Institute for Fluid Dynamics, 1997), Vol. 5.

21. S. Ta'asan, Theoretical tools for problem setup, in Inverse Design and Optimization Methods, edited by R. A. van den Braembussche and M. Manna, VKI Lecture Series (Von Karman Institute for Fluid Dynamics, 1997), Vol. 5.

22. G. D. Tzabiras, A numerical investigation of $2 \mathrm{~d}$ steady free surface flows, Int. J. Num. Meth. Fluids 25, 567 (1997).

23. M. Vogt, A numerical investigation of the level set method for computing free surface waves, Tech. Rep. CHA/NAV/R-98/0054, ISSN 1101-0614 (Chalmers Univ. Technol, 1998).

24. G. B. Whitham, Linear and Nonlinear Waves (Wiley, New York, 1974).

25. E. Zauderer, Partial Differential Equations of Applied Mathematics, 2nd ed. (Wiley, Chichester, 1989). 